Dermatologische Zeitschrift. 1921;32:I-IV

\title{
Contents, Vol. 32, 1921
}

Inhaltsverzeichnis.

Originalarbeiten. Seite

Arzt, L. , und II. Fuß, Über Sykosis parasitaria mit be-

sonderer Berücksichtigung der spezifischen Therapie . . 91

uncj W. Kerl, Über Spirochaetenbefimde im Blute von

Frühluetikern. (Hierzu Tafel VIII.) 199

und , Über die Virulenz der Spirochaete pallida

an der Infektionsstelle nach vorangegangener Therapie.

(Hierzu Tafel IX.) $\quad 32(3$

Bailer, G. , Eine neue Methode zur Vermeidung intra-

urethraler Keimverschleppung bei Kathetereinführung und

Harnröhrenspülung 39

Droop, H., Syphilisdiagnose und Drüsenpunktion .... 336

Fischer, H. , Familiar-hereditäres Vorkommen von Keratoina

pal·mare et plantare, Nagelveränderungen, Haaranomalien

und Verdickung der Endglieder der Finger und Zehen in

$\delta$ Generationen. (Die Beziehungen dieser Veränderungen

zur inneren Sekretion.) (Hierzu Tafel VII.) 114

Frieboes, W. , Beiträge zur Anatoinie und Biologie der Haut.

III. Bau des Deckepithels (II). Epithelregeneration.

Atrophien und Hypertrophien des Deckepithels. Sklero-

dermie. (Hierzu Tafel I-V.) . . 1

- $\quad$, Beiträge zur Anatomie und Biologie der Haut. IV.

Weiteres zum Rongal·itwei\&bild der Hautnerven. (Hierzu

Tafel X-XL) 267

G é b e r , H. , Einige Daten zur Pathologie der Urticaria men-

struationalis 143

Hofmann, Edm., und 0. Mergelsb ;rg, Über Allgemein-behandlung der weiblichen Gonorrhoe

mit Gonargin, Collar-gol, Silbersalvarsan, Terpentin und Trypaflavin .... 25

Kohrs, Th. , Liquorbefunde bei behandelter Syphilis ... 71

L o e w e n s t e i n, W. , Zur Frage der Sypliilisrezidive nach

Salvarsan und Neosalvarsan 223

Meirowsky, E. , Unfall als Ursache für die Entstehung von

Acrodermatitis atrophicans und Arthritis deformans . . 346

Meyer, Heinz, Experimentelle Untersuchungen über den

JY Inhaltsverzeicl·mis.

Seite

Abtransport des Hautpigments. Ein Beitrag zur Frage

des Ursprungs des Kutispigments 348 
Miescher, G. , Zwei Fälle von congenitaler Akanthosis nigricans, kombiniert mit Diabetes mellitus $27 \mathrm{G}$

Eeil, H. , Über Veränderungen der Mundschleimhaut bei

Psoriasis vulgaris 215

Kuete, A. E. , Zur Frage der Röntgentherapie bei Aktino-

mykose 344

Stühmer, A., Der klinische Verlauf der Rektalgonorrhoe.

(Mit einem Beitrag zur Fieberbehandlung der Gonorrhoe.)

(Hierzu Tafel VI.) .12

Stümpke, G. , Über die Entstehungsbedingungen der Noma 210

$\mathrm{T}$ a $\mathrm{c} h$ a u , P. , Untersuchungen über die Funktion der Leber bei Lues, unter besonderer

Berücksichtigung des Ikterus syphiliticus praecox und der Leberstörungen dureh Salvarsan 307

Gesellschaftsberichte.

Herbsttagung der rheinisch-westfälischen Dermatologenver-

einigung in Düsseldorf am 17. X. $1920 \quad 248$

Berliner Dermatologische Gesellschaft. Sitzung vom 4. III. 1920151

-, Sitzung vom 13. IV. 1920 , .... 356

Dänische Dermatologische Gesellschaft. Sitzung vom 2. IV.,

7. V. und 1. X. 1919250

Periodische Literatur 46, 169, 253, 362

Buchanzeigen 378

Tagesnachrichten und Perso $\pi$ alien ... 70, 198, 253, 378, 379

Sach- und Namenregister $\quad 380,385$ 